\title{
FORMULACIÓN DE UNA HARINA INTEGRAL \\ FUNCIONAL, A PARTIR DE CEREALES ANDINOS $Y$ SUBPRODUCTOS DE LA UVA Y EL CAFÉ
}

\author{
Sebastián Arias-Giraldo \\ Universidad Católica Luis Amigó \\ sebastian.ariasgi@amigo.edu.co. \\ Sugey Elena Anaya-García \\ Universidad Católica Luis Amigó \\ Daniel Muñoz Quintero \\ Universidad Católica Luis Amigó \\ Ana María Chaux-Gutiérrez \\ Universidad Católica Luis Amigó \\ Resumen \\ Correspondiente con el estilo de vida del ser humano, el rubro de alimentos \\ y bebidas debe ofrecer productos valiosos, con elevado aporte nutricional, \\ sostenibles, de fácil preparación y con gran durabilidad; necesidad que se \\ encuentra alineada con los Objetivos de Desarrollo Sostenible (ODS) \\ número 2, 3 y 12, planteados por la ONU. Entre las alternativas disponibles \\ en el mercado, sobresalen los concentrados, las harinas, las bebidas y los \\ comprimidos. En los últimos años, se ha buscado reutilizar los residuos de \\ diferentes sectores, aprovechando su capacidad de transformación como \\ materia prima o insumo; las industrias del café y la uva no son ajenas a esta \\ tendencia. Diversos estudios exponen el potencial de la cáscara del café y \\ la uva, como fuente de compuestos fenólicos, antioxidantes y fibra dietaria. \\ Por otra parte, la cultura gastronómica exige regresar a las raíces y a lo \\ autóctono, en congruencia con los principios de seguridad y soberanía \\ alimentaria. En los Andes, se dispone de la quinua y el amaranto, \\ caracterizados por contener la totalidad de aminoácidos esenciales; \\ además, son ricos en vitaminas $\mathrm{E}$ y $\mathrm{C}$, y calcio, hierro y zinc. El presente \\ proyecto de investigación busca formular un producto comestible integral, \\ tipo harina, que conserve los beneficios ofrecidos por la cáscara de café, el \\ epicarpio de uva, el amaranto y la quinua. Para ello, se desarrollará un \\ estudio cuantitativo experimental, siguiendo un diseño factorial con dos \\ fuentes de variación: tipo de cereal andino y clase de residuo. Como
}


respuesta, se evaluará la calidad bromatológica, antioxidante, microbiológica y sensorial del alimento, con una significancia estadística de $\mathbf{a}=0.05$. Se espera obtener un alimento aceptado por el consumidor, benéfico nutricionalmente y fácil de vincular a preparaciones gastronómicas; lo anterior, con miras a su transferencia a los actores involucrados en el servicio de alimentos y bebidas.

Palabras clave

alimento funcional, antioxidantes, gastronomía, seguridad alimentaria, subproductos.

Desde el año 2012, la definición de los retos y las prioridades del mundo ha sido objeto de una activa conversación, que da lugar a la agenda global de trabajo con horizonte al 2030. Con carácter global y de aplicabilidad universal, el Programa de las Naciones Unidas para el Desarrollo (PNUD) ha definido, dentro de los 17 Objetivos de Desarrollo Sostenible (ODS), la necesidad de priorizar los números 2, 3 y 12: poner fin al hambre, lograr la seguridad alimentaria y la mejora de la nutrición y promover la agricultura sostenible; garantizar una vida sana y promover el bienestar para todos en todas las edades; y garantizar modalidades de consumo y producción sostenibles... (Sanahuja, 2015). Lo expuesto anteriormente, desde la perspectiva del sector gastronómico, demanda la creación de nuevas formas de alimentarse, de manera que estas alternativas sean asequibles, nutritivas, económicas, sustentables y sostenibles.

Es evidente que el estilo de vida contemporáneo provoca un fuerte impacto en los hábitos alimentarios, con un consumo creciente de productos rápidos y altamente procesados, cuyos efectos adversos sobre la salud son claramente perceptibles. La comida en el mundo occidental está asociada al placer, de modo que el compromiso entre la gratificación y la salud es incierto. Los problemas de salud asociados a la ingesta de 
alimentos: diabetes, cáncer, fallas cardíacas y obesidad, afectan por igual a adultos y niños, con consecuencias visibles en países en vía de desarrollo, donde los efectos nocivos resultan más evidentes que en países del primer mundo (Ezzati et al., 2015).

Para el año 2016, el 21\% del mercado mundial de alimentos correspondió a productos con proclamas sobre salud y bienestar, generando movimientos financieros cercanos a los 426.000 millones de dólares. Fueron relevantes, en su orden, matrices que contribuyen al bienestar general, el control de peso y la salud digestiva. Para Colombia, el 19\% del mercado alimentario está asociado a productos funcionales y nutracéuticos, evidenciando un incremento anual promedio del 12\% (Corporación Biointropic, 2018).

Las oportunidades de los alimentos funcionales en el campo de la salud y el bienestar son inmensas. Pueden contribuir a mejorar la salud cardiaca, mantener una presión sanguínea adecuada, asegurar condiciones cognitivas, potenciar el aprovechamiento energético, fortalecer huesos y articulaciones, mantener una piel saludable, ayudar en el balance de la dieta y el control de peso, entre otros (FuentesBerrío et al., 2015).

Actualmente, existe una oferta limitada de harinas funcionales, siendo comunes únicamente ingredientes como el arroz, el maíz y la soya, los cuales exhiben el inconveniente de afectar las propiedades reológicas y texturales del pan y otras preparaciones (Hernández et al., 2018). Los productos autóctonos colombianos tienen una baja participación en este rubro. 
La quinua y el amaranto son pseudocereales andinos, de fácil acceso en Colombia. Destacan, en su conjunto, la presencia de todos los aminoácidos esenciales, proteínas de gran calidad biológica, fibra dietaria, vitaminas $\mathrm{E}, \mathrm{B}$ y $\mathrm{C}$, y minerales como fósforo, potasio, calcio, hierro y zinc. Ambas materias primas se pueden utilizar en el reemplazo parcial, o total, de harinas tradicionales, con usos en panadería, repostería, conservas, helados, dulces y bebidas (Blasco \& Gómez, 2014; Hernández, 2015; Dyner et al., 2018).

La mayor parte de los alimentos saludables se vincula a la industria láctea (prebióticos y probióticos), siendo también destacables los rubros de panadería, alimentos infantiles, confites y refrescos (Illanés, 2015). Así, se hace necesario evaluar la funcionalidad de otras materias primas alimentarias, como los cereales, las frutas y las hortalizas; las cuales, de manera integral, poseen un sin número de nutrientes y componentes potencialmente benéficos para la salud. Estos alimentos poseen propiedades que vinculan, incluso, las semillas, cáscaras y otros elementos que, tradicionalmente, son dispuestos como residuos.

Una tercera parte de los alimentos que se producen en el mundo acaban en la basura, dando lugar a una tasa de desperdicio, por habitante del planeta, de 177 kg/año. La disposición inadecuada de los residuos agroalimentarios, sumada a su nulo aprovechamiento como subproductos, causan el $8 \%$ de las emisiones globales de efecto invernadero (Revista Semana, 2019).

En los últimos años, se ha sugerido el uso de los subproductos del café como fuentes de sustancias químicas funcionales y bioactivas, entre las que se encuentran los polifenoles y la cafeína, de gran utilidad para la 
industria alimenticia y farmacéutica (Esquivel \& Jiménez, 2012). Puertas-Mejía et al. (2013) han demostrado que la borra del café presenta capacidad secuestrante de radicales libres. Por su parte, la cáscara y las semillas de la uva son consideradas fuentes importantes de compuestos fenólicos, a los que se les atribuyen las propiedades astringentes y antioxidantes de sus productos derivados (Sato et al., 2001). Algunos reportes indican que la funcionalidad de la uva, así como la capacidad antioxidante total de la matriz, depende significativamente de la especie, tipo y tejido del fruto, siendo necesaria la evaluación específica de los efectos benéficos que ofrecen la pulpa, la semilla y el epicarpio (Molina et al., 2010).

De acuerdo con lo expuesto anteriormente, el presente proyecto responderá la siguiente pregunta de investigación: ¿cuál es la mejor formulación para una harina integral con potencial funcional, que involucre la quinua, el amaranto, la cáscara de café y el epicarpio de uva como ingredientes, de manera que se favorezcan sus atributos fisicoquímicos, nutricionales y sensoriales?

\section{Marco teórico}

\section{Harinas y sector harinero}

La definición de harina se asocia al proceso de molienda que se aplica al grano de trigo; el núcleo del grano se separa en tres partes y, según el aprovechamiento que se le da a cada una de ellas, recibe un nombre distinto al comúnmente conocido como harina de trigo (Forkish, 2019). Sin embargo, el Larousse Gastronomique (2011), 
define harina como producto de la molienda de cualquier cereal, vegetal - leguminosa. Esto indica que los ingredientes se pueden considerar harina, si se les aplica un procesado adecuado y se declara la materia prima de la cual proviene.

Es importante tener en cuenta que para definir una harina como integral, según la Norma Técnica Colombiana (NTC) 5945, estructurada por el ICONTEC (2012), el producto que se obtiene de la pulverización debe conservar el pericarpio, endospermo y germen. Por otro lado, un alimento es considerado funcional si en su consumo ordinario y constante, puede mejorar el estado de salud o aminorar los riesgos de enfermedad. Varias organizaciones aceptan el término funcional cuando el alimento demuestra un beneficio fisiológico distinto al que de manera común ofrece nutricionalmente. Se ha evidenciado un gran crecimiento en la oferta de alimentos funcionales en el mercado, los consumidores son más conscientes y tiene más interés por la promoción de dietas saludables. El desafío para estos alimentos es cada vez mayor, deben ser nutritivos, sanos y agradables al ser consumidos (Morais et al., 2018).

Las expectativas para el sector productor de cereales y leguminosas en Colombia son muy favorables. Desde el 2018, el sector agropecuario ha tenido un crecimiento en la siembra de estos ingredientes, las importaciones de granos han disminuido y todo esto se ha logrado con el uso de semillas que tienen mayor productividad por hectárea y mayor resistencia a los cambios climáticos. Como lo afirma la Revista Portafolio (2018), el sector de alimentos y bebidas alcanzó ventas por 13.200 millones de dólares en 2017 y tuvo más presentación en las industrias de molinería, panadería y repostería, con un 31\%; seguido de los lácteos, 
harinas, confitería, snacks, frutas, legumbres, aceites, salsas, cárnicos, helados y postres. El sector harinero, se ha visto en la tarea de aprovechar otro tipo de cereales y leguminosas para diversificar la oferta de productos, ya que el costo de las harinas de trigo ha incrementado notablemente (Olaya, 2019).

Materias primas alternativas, focalizadas para la fabricación de la harina integral

Es clara la importancia del desarrollo de nuevos productos alimenticios que contribuyan, a su vez, con las tendencias actuales y venideras para el sector gastronómico: (a) mejores procesos, técnicas, productos y equipos, (b) productos ancestrales, auténticos y locales, (c) sentido y entorno social, y (g) salud y nutrición (Fondo Nacional de Turismo, 2017). Atendiendo estas directrices, en la presente propuesta de investigación se utilizarán dos pseudocereales autóctonos de los Andes, quinua y amaranto, los cuales demandan recuperación en su consumo y, además, ofrecen un gran valor nutritivo y sensorial. Por otra parte, el compromiso con el medio ambiente y el entorno social se ven caracterizados al utilizar residuos de las industrias vitivinícola y del café, ambos de gran protagonismo en Colombia.

Quinua

La quinua ha existido desde épocas muy antiguas; sin embargo, hasta hace poco su popularidad ha incrementado alrededor del mundo, incluyendo Colombia. La quinua (Chenopodium quinoa) es una planta dicotiledónea, cultivada mayormente en Sudamérica, que 
suele ser consumida de la misma manera que un cereal, de ahí que se le denomine pseudocereal (FAO, 2014).

Morillo et al. (2017), mencionan que la quinua es considerada un cereal con excelentes propiedades nutricionales, entre las que se destacan su alto contenido proteico, ya que tiene todos los aminoácidos y cantidades significativas de vitaminas C, E (tocoferoles) y B (B1, B2 y B3), junto con importantes minerales $(\mathrm{Ca}, \mathrm{K}, \mathrm{Fe}, \mathrm{Mg}, \mathrm{Mn}, \mathrm{P})$ e isoflavonas, que pueden contribuir a sus propiedades antioxidantes. La quinua no tiene gluten y sus ácidos grasos son de alta calidad.

En informe técnico de la FAO (2011), se establece que la quinua cuenta con más de tres mil variedades, incluidas las silvestres. Además, su cultivo es adaptable a diferentes pisos térmicos y su crecimiento se puede dar en rangos de humedad entre $40 \%$ - $88 \%$, soportando temperaturas que van entre $-4^{\circ} \mathrm{C}$ a $38^{\circ} \mathrm{C}$.

La quinua es un producto altamente aprovechable, teniendo en cuenta sus usos en diferentes industrias: tallos para concentrados y obtención de papel y celulosa, y hojas para la elaboración de ensaladas, harinas y colorantes. Desde la perspectiva agroindustrial, el grano perlado se utiliza en panes, galletas, salsas, fideos, granolas, sopas, snacks, extruidos, postres y dulces. Por su alta fracción saponificable, el residuo de lavado de esta materia prima se puede usar en la fabricación de champú, detergentes, pasta dental y pesticidas (Montoya et al., 2005).

La quinua, para poder ser consumida, debe pasar por muchos procesos, como el lavado, secado y cocción; siendo este último uno de los que más afecta la conservación del contenido nutrimental (Mendoza et al., 2015). 
Amaranto

El amaranto (Amaranthus spp.) proviene de la región andina de Suramérica, consumiéndose como hortaliza y como cereal. Este alimento, pseudocereal, no contiene gluten (FAO, 2019). Debido al aporte vitamínico y los pigmentos que el amaranto proporciona, se pueden elaborar helados, postres, harinas, entre otros productos; teniendo en cuenta, además, que por su contenido de polifenoles y compuestos bioactivos se constituye como una fuente de antioxidantes, importantes en la prevención del cáncer (Herrera \& Montenegro, 2012).

El amaranto es uno de los granos que más contenido proteico posee, principalmente asociado a aminoácidos como lisina, metionina y cisteína. La secuencia de péptidos en su estructura muestra una actividad biológica benéfica para la salud (González et al., 2013).

\section{Cáscara de café}

Los climas y suelos de Colombia son el conjunto perfecto para que la especie de café, Coffea arábica, crezca abundantemente en la mayoría de las regiones colombianas. La producción de este grano incrementa anualmente y se ha convertido en un motor económico, además de ser el sector que más ha integrado socialmente a los colombianos (Arcila et al., 2007).

El principal subproducto del aprovechamiento de café es la cáscara o epicarpio, fácil de identificar por su coloración roja cuando se recoge en estado de maduración óptimo. La cáscara y el mucílago del café 
representan el $61 \%$ de la materia fresca de la cereza; siendo los principales contaminantes de las cuencas y/o terrenos agrícolas. Uno de los usos que se le ha dado al epicarpio de café, se relaciona con la alimentación de ganado. Sin embargo, se desconoce su contenido apreciable de carbohidratos, proteínas y minerales, además de antioxidantes como taninos, cafeína y polifenoles (Figueroa et al., 2015).

Los subproductos del café muestran registros capacidad antioxidante. Estos residuos, con su respectivo tratamiento, podrían emplearse como una fuente natural de compuestos fenólicos. El ácido clorogénico es uno de los que más abunda en el café; ha sido asociado con la reducción del riesgo de padecer: diabetes tipo dos, cirrosis hepática, cáncer de hígado y colon, enfermedades cardiovasculares y neurodegenerativas (Fonseca et al., 2014).

\section{Cáscara de uva}

La uva (Vitis vinífera) es un fruto comestible, que crece en la vid, excelente como recuperador energético. Contiene vitaminas B1, B6 y C, además de minerales como hierro y potasio. Es la materia prima para la fabricación del vino, proceso en el cual se aprovechan todas sus partes, con distintas finalidades (Ordoñez et al., 2019).

La producción de vino genera residuos que la mayoría de las empresas no aprovechan, al no identificar su valor potencial. Los principales desperdicios que deja este proceso son semillas, cáscaras y fibras, los cuales incluyen entre sus componentes antioxidantes, que pueden favorecer el cuidado y preservación de la salud (Sandoval et al., 2008). En 
promedio, el $20 \%$ de la uva acaba como deshecho de la producción de vino. Para minimizar este fenómeno, se han realizado investigaciones en las que se puede obtener harina de uva (Sainz et al., 2019).

Las uvas contienen gran cantidad de compuestos fenólicos en la cáscara y, específicamente, en las células epidérmicas se encuentran los flavonoles y antocianos. A estos se les atribuye funciones como agentes vasodilatadores, antiinflamatorios y anticancerígenos (Sandoval et al., 2008). Se ha encontrado que las uvas rojas tienen mayor contenido de flavonoides y que la concentración de estos antioxidantes puede variar según la maduración del fruto. Sin embargo, independientemente de la variedad de uva y estado de maduración, los niveles de polifenoles que presentan son similares (Molina et al., 2010).

Composición nutricional de las materias primas seleccionadas

La Tabla 1 presenta, de manera ordenada, la composición reportada para cada materia prima.

Tabla 1. Composición promedio, según diferentes autores, para las cuatro materias primas focalizadas.

\begin{tabular}{|c|c|c|c|c|c|c|}
\hline $\begin{array}{c}\text { Matriz vegetal } \\
\text { Nutriente }^{*}\end{array}$ & Quinua* & Amaranto $^{* *}$ & Uva negra ${ }^{* \star \star}$ & $\begin{array}{c}\text { Café } \\
\text { tostado }^{* *}\end{array}$ & $\begin{array}{l}\text { Cáscara de } \\
\text { café }^{\star \star \star *}\end{array}$ & $\begin{array}{c}\text { Cáscara de } \\
\text { uva }^{* \star *}\end{array}$ \\
\hline Proteína (g) & 14,1 & $12,0-19,0$ & 0,6 & 12,6 & 11,5 & 11,5 \\
\hline Carbohidratos (g) & 64,2 & 71,8 & 17,2 & 63,4 & - & - \\
\hline Lípidos (g) & 6,1 & $6,1-8,1$ & 0,7 & 14,8 & 2,0 & 12,4 \\
\hline Fibra (g) & 7,0 & $3,5-5,0$ & 0,9 & - & 60,5 & - \\
\hline Cenizas (g) & 2,4 & $3,0-3,3$ & 0,6 & 4,8 & - & 4,3 \\
\hline Energía (kcal) & 368 & 391 & 67 & 226 & - & - \\
\hline Calcio (mg) & 47 & $130-164$ & 14 & 148 & - & - \\
\hline Fósforo (mg) & 457 & 530 & 10 & 198 & - & - \\
\hline Potasio (mg) & 563 & 800 & 191 & - & - & - \\
\hline
\end{tabular}




\begin{tabular}{|c|c|c|c|c|c|c|}
\hline Referencia & $\begin{array}{c}\text { Bazile et al. } \\
(2014)\end{array}$ & Nieto (1990) & $\begin{array}{l}\text { INCAP } \\
(2012)\end{array}$ & $\begin{array}{l}\text { INCAP } \\
(2012)\end{array}$ & $\begin{array}{c}\text { Figueroa et } \\
\text { al. (2015) }\end{array}$ & $\begin{array}{c}\text { Sainz et al. } \\
(2019)\end{array}$ \\
\hline
\end{tabular}

\section{Metodología}

\section{Proceso de conceptualización y revisión bibliográfica}

Se realizará una revisión bibliográfica estructurada, utilizando diferentes fuentes y referentes de carácter técnico y científico. Se usarán artículos de investigación publicados en revistas indexadas, libros de divulgación e investigación, trabajos de grado de especialización y maestría, tesis doctorales, normas técnicas y regulaciones (nacionales e internacionales), manuales de laboratorios reconocidos, bases de datos de patentes (Espacenet, WIPO Patentscope, Super Intendencia de Industria y Comercio de Colombia SIC), documentos emitidos por entidades gubernamentales oficiales, y revistas no académicas, especializadas en temas económicos, industriales y agroalimentarios.

La consulta, organización y análisis de la información focalizada, se simplificará por medio del acceso a bases de datos licenciadas por la Universidad Católica Luis Amigó. Se tendrán en cuenta, entre otras: Elibro, Digitalia, EBSCO, Legismóvil, Oxford, Science Direct, Scopus, Springer y Scielo. En total, se revisarán un mínimo de 50 referencias, plasmadas en una estrategia de sistematización que dé cuenta de las temáticas principales asociadas al desarrollo del proyecto de investigación. 
La revisión bibliográfica conllevará una pesquisa activa de índole teórico, relacionada con los siguientes descriptores: desarrollo de nuevos productos, secado y deshidratación de alimentos, análisis de alimentos, nutrición humana, amaranto, quinua, uva, epicarpio de uva, café, cáscara de café, optimización de procesos, análisis sensorial, antioxidantes, aprovechamiento de residuos, harinas, agroindustria, alimentos funcionales, proteínas, fibra dietaria, entre otros.

Fabricación de cada una de las harinas y evaluación de condiciones de procesamiento

Materia prima

La quinua, el amaranto, la cáscara de café y el epicarpio de uva, como materias primas del producto a formular serán adquiridas siempre con un mismo proveedor. Lo anterior, puede hacer referencia a una empresa (tienda o supermercado), o a la negociación directa con un productor de la ciudad de Medellín o la región Antioquia, Eje cafetero. El encargado del aprovisionamiento garantizará la uniformidad de los productos suministrados, en aspectos como estado de madurez, color, aspecto visual, tamaño, aroma y sabor.

Para cada materia prima cruda y húmeda, se adquirirá un lote de mínimo $15 \mathrm{~kg}$ por experimento. En caso de ser necesario el transporte del material vegetal, desde una ciudad diferente a Medellín, el desplazamiento se hará bajo condiciones de refrigeración. La materia prima se mantendrá a una temperatura entre $4^{\circ} \mathrm{C}$ a $7^{\circ} \mathrm{C}$. De igual forma, se respetará este rango de temperatura para el 
almacenamiento de los productos en las instalaciones de la Universidad Católica Luis Amigó, hasta el día de las pruebas para la fabricación de las harinas.

\section{Alistamiento de materias primas}

La materia prima se lavará inicialmente con abundante agua, con el fin de retirar material extraño y sólidos contaminantes. Posteriormente, se realizará la respectiva sanitización del material vegetal, utilizando desinfectante orgánico a base de ácido láctico, marca Suma Edén. Cada producto se llevará a inmersión durante 5 minutos, en una solución de 1 $\mathrm{ml}$ de reactivo en 1 I de agua. Finalmente, se revisará el estado general de cada materia prima, para proceder a su secado superficial utilizando un cedazo.

La quinua pasará, adicionalmente, por un proceso de desaponificación, el cual consiste en llevar a cabo lavados consecutivos de los granos con agua caliente $\left(50^{\circ} \mathrm{C}\right)$, bajo agitación constante, hasta que cese la formación de espuma (Repo et al., 2011).

Procesamiento de las materias primas focalizadas y elaboración de harinas

Para la fabricación de las harinas, se seguirán parcialmente los protocolos propuestos por Abarca et al. (2010), Molina et al. (2010) y Repo et al. (2011). Todos los ensayos de transformación, para cada una 
de las materias primas, se realizarán en los laboratorios de cocina de la Universidad Católica Luis Amigó.

La deshidratación de las materias primas, como operación crítica en la elaboración de harinas, se analizará según un diseño experimental factorial completamente al azar, con dos fuentes de variación: tipo de material vegetal y temperatura de secado. El primer factor, evaluará cuatro niveles: epicarpio de uva, cáscara de café, amaranto y quinua; mientras que el segundo factor considerará dos niveles: $55^{\circ} \mathrm{C}$ y $70^{\circ} \mathrm{C}$. En total, esta etapa del proyecto incluirá ocho tratamientos, ejecutados por duplicado, dando lugar a un total de 16 experimentos. La variable de respuesta corresponderá a la estimación de atributos sensoriales, para cada uno de los polvos obtenidos.

Con miras al secado de los productos, se utilizará un horno de convección combinado marca Rational, modelo SCC1016 (Self Cooking Center). Se hará seguimiento de la temperatura real del aire caliente, empleando un termopar tipo K (Hanna HI766C) asociado a termómetro digital (Hanna HI93531N). Cada 10 min, se pesará la bandeja que contiene el material vegetal en deshidratación, utilizando una balanza de precisión Trúmax, modelo MIX-A. Lo anterior, con el fin de construir las respectivas curvas y cinéticas de remoción de agua. El proceso de secado tendrá lugar hasta alcanzar peso constante en las matrices de interés (humedad mínima del 8\%).

Las cinéticas de secado se analizarán utilizando el programa Microsoft Excel, determinando los siguientes puntos de la operación: tasa constante y decreciente de deshidratación, y humedad crítica y de equilibrio. 
Los insumos deshidratados se llevarán a un procesador de alimentos Waring WFP 16, con el fin de reducir su tamaño individual de partícula. Se hará control del diámetro medio del polvo, hasta alcanzar un valor de $210 \mu \mathrm{m}$, equivalente a una malla 65 de Tyler - 70 US Standard (ICONTEC, 2007a).

Finalmente, las muestras de harina se empacarán al vacío y de forma independiente, en un equipo marca Komet Vacuboy. Los productos de cada matriz vegetal se almacenarán en un lugar seco y oscuro, hasta el día en que proceda su análisis de calidad.

La evaluación sensorial, para cada una de las harinas, seguirá los procedimientos y las condiciones de prueba establecidas por AnzaldúaMorales (1994). Se utilizarán un total de 20 jueces semientrenados, que llevarán a cabo los siguientes ensayos: (a) prueba afectiva, con escala hedónica verbal de 5 puntos, y (b) prueba discriminativa de tipo triangular.

Para cada harina elaborada, se evaluarán los siguientes atributos tecnológicos: solubilidad, capacidad de formación y estabilidad de espuma.

El polvo con los mejores atributos sensoriales y tecnológicos, proveniente de cada matriz vegetal, se considerará como insumo potencial del producto final a formular. La selección de la mejor harina tendrá en cuenta la comparación de las propiedades de los diferentes tratamientos. Para ello, se usará el software Statgraphics Centurion XVI, mediante un ANOVA con $\mathbf{a}=0.05$. Además, se estructurará una prueba pareado de Tukey, previa validación de los supuestos de normalidad y homocedasticidad. 
Elección de la mejor formulación, que integre las materias primas focalizadas

Contando con las mejores harinas, obtenidas a partir de cada materia prima, se procederá a preparar las muestras formuladas del producto final. Se seguirá un diseño experimental factorial completamente al azar, con dos fuentes de variación: clase de cereal andino (quinua o amaranto) y tipo de subproducto (cáscara de café o epicarpio de uva). Con dos niveles por factor, se tendrá un total de cuatro tratamientos, evaluados por duplicado (8 experimentos).

Cada tratamiento corresponderá a la mezcla, en proporciones iguales (50/50), de harinas provenientes de un cereal andino y de un subproducto. Las muestras se homogenizarán, para ser empacadas nuevamente en la máquina Komet, modelo Vacuboy. Las muestras se enviarán para análisis de laboratorio, por triplicado, considerando los parámetros presentados en la Tabla 2:

Tabla 2. Pruebas de laboratorio, para el análisis de la harina integral formulada.

\begin{tabular}{|c|c|c|}
\hline Categoría & Prueba & Norma, protocolo o referencia \\
\hline \multirow[t]{5}{*}{ Caracterización bromatológica * } & Humedad & AOAC 934.16 \\
\hline & Proteínas & AOAC 920.152 \\
\hline & Lípidos & AOAC 945.39 \\
\hline & Fibra & AOAC 991.43 \\
\hline & Cenizas & AOAC 940.26 \\
\hline \multirow[t]{2}{*}{ Capacidad antioxidante } & Método ABTS & Arnao (2000) \\
\hline & Contenido de fenoles totales & \\
\hline \multirow[t]{3}{*}{ Análisis microbiológico ** } & Mesófilos aerobios & NTC 4519 \\
\hline & Coliformes totales & NTC 4458 \\
\hline & Hongos y levaduras & NTC 4132 \\
\hline
\end{tabular}

El análisis estadístico de los resultados obtenidos, para cada una de las cuatro formulaciones de harina, se llevará a cabo por medio de un ANOVA con $\mathbf{a}=0.05$, seguido de una prueba de múltiples rangos de Tukey. En todos los casos, se validarán los supuestos de normalidad y 
homocedasticidad. Para el procesamiento de datos, se utilizará en todos los casos el software Statgraphics Centurion XVI.

La mejor formulación para la harina integral se elegirá a través de un análisis de sensibilidad, que considerará la totalidad de los factores evaluados en la calidad del producto: atributos fisicoquímicos, aspectos microbiológicos, capacidad antioxidante y propiedades tecnológicas.

\section{Validación de la harina integral en procesos gastronómicos}

Para la formulación elegida, se validará su uso en diferentes líneas gastronómicas. Se deberán considerar propiedades tecnológicas como capacidad de hinchamiento, retención de agua, atributo espumante, gelificación, poder espesante y solubilidad. Por medio de la observación del comportamiento de las harinas, más el análisis de la composición final del alimento, se probará su aptitud para elaborar masas friables, batidos livianos, panes, coladas, bebidas, empanizados, u otros que se consideren pertinentes. Como mínimo, se sugerirá un uso para el producto final.

\section{Resultados e impactos esperados}

El proyecto de pesquisa corresponde al nivel de investigación aplicada, generando como principal resultado un producto alimenticio formulado. La particularidad de la harina que se desea obtener radica en la utilización de subproductos del café y la uva, así como la vinculación de dos cereales andinos de alto valor tecnológico: amaranto y quinua. 
Así, se dará inicio al proceso de desarrollo de una harina con gran potencial nutricional, destacándose la concentración de proteínas, antioxidantes, vitaminas y minerales. De esta manera, a futuro, se podrá impactar positivamente la salud de los consumidores del alimento propuesto.

A través de la publicación y socialización de los resultados de investigación, se favorecerá la participación de la Universidad Católica Luis Amigó en nuevos escenarios y entornos académicos. Además, al tratarse de una propuesta que puede escalarse a nivel industrial y/o comercial, podrá dar lugar al establecimiento de alianzas y convenios con otras instituciones y el sector productivo. Por otra parte, la propuesta contribuirá a la consolidación del programa de Gastronomía, del grupo de investigación Goras y de la Línea de Investigación e Innovación en Ciencia, Tecnología y Cultura Gastronómica. En el marco de este proyecto, también se desarrollará la Beca Pasantía de una joven investigadora y las actividades de tres auxiliares de investigación, afianzando la investigación formativa de la Institución.

Los resultados de investigación de la presente propuesta podrán ser de utilidad para el sector alimenticio y gastronómico, enfocado hacia la transformación positiva de materias primas agroindustriales. Se reportarán condiciones ideales de procesado, que pueden asociarse a otro tipo de matrices o productos finales. Así, este proyecto podrá ser el punto de partida para nuevas y mejores investigaciones, que busquen ofrecer al consumidor más alimentos, de 
calidad, más rentables, aprovechando la integralidad de los insumos utilizados.

La presente investigación entregará una opción de producto alimenticio, que vinculará en su formulación la utilización de por lo menos un subproducto de importancia para el país. De esta manera, se podrá apalancar el aprovechamiento de residuos en algunos procesos de transformación, alargando el ciclo de vida de las materias primas vegetales focalizadas: café y uva. Así, a futuro, se espera que la experiencia sirva como ejemplo a los sectores académico, científico y productivo, buscando la implementación a escala real del mejor proceso de fabricación.

A través de los productos generados, se buscan analizar y divulgar diferentes alternativas viables para el aprovechamiento de los residuos del sector cafetero y vitivinícola. Así, se podrá contribuir a la disminución en las tasas de desperdicio de estas dos industrias, una vez sean implementadas algunas de las estrategias sugeridas en el desarrollo del trabajo de pesquisa.

\section{Conclusiones}

Al tratarse de una propuesta de investigación, aún no hay conclusiones derivadas de los resultados. Se espera, al finalizar la ejecución del proyecto, concluir sobre la pertinencia y la oportunidad de la harina funcional desarrollada. 


\section{Agradecimientos}

A la Universidad Católica Luis Amigó, quién apoya y financia el desarrollo del presente proyecto de investigación.

\section{Referencias}

Abarca, D., Martínez, R., Muñoz, J., Torres, M. \& Vargas, G. (2010). Residuos de café, cacao y cladodio de tuna: fuentes promisorias de fibra dietaria. Revista Tecnológica ESPOL RTE, 23 (2), 63-69.

Anzaldúa-Morales, A. (1994). La evaluación sensorial de los alimentos en la teoría y la práctica. Acribia.

AOAC. (2004). Official method of analysis of the association of official analytical chemists. 15 Ed Association of Official Analytical Chemists (AOAC).

Arcila, P.J., Farfán, V., Moreno, B., Salazar, G. \& Hincapié G. (2007). Sistemas de Producción de Café en Colombia. Centro Nacional de Investigación en Café (Cenicafé).

Arnao, M. B. (2000). Some methodological problems in the determination of antioxidant activity using chromogen radicals: a practical case. Trends in Food Science, 11, 419421.

Bazile, D., Bertero, D. \& Nieto, C. (2014). Estado del arte la quinua en el mundo en 2013. Organización de las Naciones Unidas para la Alimentación y la Agricultura (FAO) Centro de Cooperación Internacional en Investigación Agronómica para el Desarrollo (CIRAD).

Blasco, G. \& Gómez, F.J. (2014). Propiedades funcionales del plátano (Musa sp). Revista médica Universidad Veracruzana, 14 (2), 22-26.

Corporación Biointropic. (2018). Estudios sobre bioeconomía, como fuente de nuevas industrias basadas en el capital natural de Colombia, fase II: Análisis de la situación y recomendaciones de política de bioeconomía. Anexo II: Análisis sector alimentos y bebidas. Editorial Universidad EAFIT.

Dyner, L., Drago, S.R., Piñeiro, A., Sánchez, H., González, R., Villamil, E. \& Valencia, M.E. (2018). Composición y aporte nutricional de hierro, calcio y zinc de panes y fideos elaborados con harinas de trigo y amaranto. Archivos Latinoamericanos de Nutrición, 57 (1), 69-77.

Esquivel, P. \& Jiménez, V.M. (2012). Functional properties of coffee and coffee by-products. Food Research International, 46, 488-495.

Ezzati, M., Obermeyer, Z., Tzoulaki, I., Mayosi, B.M., Elliot, P. \& León, D.A. (2015). Contributions of risk factors and medical care to cardiovascular mortality trends. Nature 
Reviews Cardiology, 12, 508-530.

FAO. (2011). La quinua: cultivo milenario para contribuir a la seguridad alimentaria mundial. Organización de las Naciones Unidas para la Alimentación y la Agricultura (FAO).

FAO. (2014). Recetario internacional de la quinua: tradición y vanguardia. Organización de las Naciones Unidas para la Alimentación y la Agricultura (FAO).

FAO. (2019). Cultivos tradicionales: amaranto. Organización de las Naciones Unidas para la Alimentación y Agricultura (FAO). http://www.fao.org/traditional-crops/amaranth/es/

Figueroa, E., Pérez, F. \& Godínez, L. (2015). La producción y el consumo del café. ECORFAN. Fondo Nacional de Turismo. (2017). Estudio sobre gastronomía colombiana a nivel nacional e internacional, y evaluar la gastronomía regional de la Guajira como producto para potenciar el turismo. Ministerio de Comercio, Industria y Turismo de Colombia.

Fonseca, L., Calderón, L.S. \& Rivera, M.E. (2014). Capacidad antioxidante y contenido de fenoles totales en café y subproductos del café producido y comercializado en Norte de Santander (Colombia). Vitae, 21 (3), 228-236.

Forkish, K. (2019). Flour water salt yeast: the fundamentals of artisan bread and pizza. Ten Speed Press.

Fuentes-Berrío, L., Acevedo-Correa, D. \& Gélvez-Ordoñez, V.M. (2015). Alimentos funcionales: impacto y retos para el desarrollo y bienestar de la sociedad colombiana. Biotecnología en el sector agropecuario y agroindustrial, 13 (2), 140-149.

González, J.E., Franco, R., Carrizales, R. \& Martínez, J.L. (2013). Perspectivas de nuevos productos a base de amaranto: Cerveza artesanal de amaranto. Tlatemoani: Revista Académica de Investigación, 14, 1-23.

Hernández, J. (2015). La quinua, una opción para la nutrición del paciente con diabetes mellitus. Revista Cubana de Endocrinología, 26 (3), 304-312.

Hernández, G., Álvarez, M. \& Rosas, B. (2018). Caracterización reológica de mezclas de harina de trigo y una premezcla fortificada de maíz y soya. Ciencia y Tecnología de Alimentos, 28 (1), 41-44.

Herrera, S. \& Montenegro, A. (2012). El amaranto: prodigioso alimento para la longevidad y la vida. Tendencias Gastronómicas, 8, 50-66.

ICONTEC. (1997). Norma Técnica Colombiana - NTC - 4132: Microbiología. Guía general para el recuento de mohos y levaduras. Técnica de recuento de colonias a $25^{\circ} \mathrm{C}$. Instituto Colombiano de Normas Técnicas y Certificación (ICONTEC).

ICONTEC. (2007a). Norma Técnica Colombiana - NTC - 267: Harina de trigo. Instituto Colombiano de Normas Técnicas y Certificación (ICONTEC).

ICONTEC. (2007b). Norma Técnica Colombiana - NTC - 4458: microbiología de alimentos y de alimentos para animales. Método horizontal para el recuento de coliformes o escherichia coli o ambos. Técnica de recuento de colonias utilizando medios fluorogénicos o cromogénicos. Instituto Colombiano de Normas Técnicas y Certificación (ICONTEC).

ICONTEC. (2009). Norma Técnica Colombiana - NTC - 4519: Microbiología de los alimentos para consumo humano y animal. Método horizontal para el recuento de microorganismos. Técnica de recuento de colonias a $30^{\circ} \mathrm{C}$. Instituto Colombiano de Normas Técnicas y Certificación (ICONTEC). 
ICONTEC. (2012). Norma Técnica Colombiana - NTC - 5945: Harina integral de trigo. Instituto Colombiano de Normas Técnicas y Certificación (ICONTEC).

Illanés, A. (2015). Alimentos funcionales y biotecnología. Revista Colombiana de Biotecnología, 17 (1), 5-8.

INCAP. (2012). Tabla de composición de alimentos de Centroamérica. Instituto de Nutrición de Centroamérica y Panamá (INCAP) - Organización Panamericana de la Salud (OPS).

Larousse. (2011). Larousse Gastronomique. Larousse Editorial.

Mendoza, E.J., Díaz, G.L. \& Vidaurre, J.M. (2015). Cinética de la degradación de betalainas y fenoles totales durante la cocciónn de la quinua (Chenopodium quinoa). Revista Ingeniería: Ciencia, Tecnología e Innovación, 2 (2), 85-95.

Molina, D.M.A., Medina, L.A., González, G.A., Robles, R.M. \& Gámez, N. (2010). Compuestos fenólicos y actividad antioxidante de cáscara de uva (Vitisvinifera L.) de mesa cultivada en el noroeste de México. CyTA - Journal of Food, 8 (1), 57-63.

Montoya, L.A., Martínez, L. \& Peralta, J. (2005). Análisis de variables estratégicas para la conformación de una cadena productiva de quinua en Colombia. Innovar, 2005, 103 119.

Morais, B., Lumy, P. \& Vannucchi, H. (2018). Efecto del resveratrol, la catequina y el gingerol en la obesidad: evidencias incompletas. Nutrición Clínica en Medicina, 12 (1), 37-46.

Morillo, A.C., Castro, M.A. \& Morillo, Y. (2017). Caracterización de la diversidad genética de una colección de quinua (Chenopodium quinoa Willd). Biotecnología en el Sector Agropecuario y Agroindustrial, 15 (2), 49-56.

Nieto, C. (1990). El cultivo de amaranto (Amaranthus spp) una alternativa agronómica para Ecuador. Publicación Miscelánea N52. Instituto Nacional de Investigaciones Agropecuarias del Ecuador (INIAP).

Olaya, M. (2019). Sube el precio del plan por alto costo de insumos. La FM. https://www.lafm.com.co/economia/sube-el-precio-del-pan-por-alto-costo-deinsumos

Ordoñez, E.S., León-Arévalo, A., Rivera-Rojas, H. \& Vargas, E. (2019). Cuantificación de polifenoles totales y capacidad antioxidante en cáscara y semilla de cacao (Theobroma cacao L.), tuna (Opuntia ficus indica Mill), uva (Vitis Vinífera) y uvilla (Pourouma cecropiifolia). Scientia Agropecuaria, 10 (2), 175-183.

Puertas-Mejía, M.A., Villegas-Guzmán, P. \& Alberto-Rojano, B. (2013). Borra de café colombiano (Coffea arabica) como fuente potencial de sustancias con capacidad antirradicales libres in vitro. Revista Cubana de Plantas Medicinales, 18 (3), 469-478.

Repo, R., Julio, J. \& Encina, C.R. (2011). Desarrollo y elaboración de un snack extruido a partir de quinua (ChenopodiumquinoaWilld.) y maíz (Zea mays L.). Ingeniería Industrial, 29, 209-224.

Revista Portafolio. (2018). Industria de alimentos y bebidas tendría un crecimiento anual del 7\%. Portafolio. https://www.portafolio.co/negocios/bajaria-ritmo-de-promociones-enlos-supermercados-del-pais-541539

Revista Semana. (2019). 12 innovaciones para acabar con el desperdicio de comida. Semana Sostenible. $\quad$ https://sostenibilidad.semana.com/medio-ambiente/articulo/12innovaciones-para-acabar-con-el-desperdicio-de-comida/43964 
Sainz, R.L., Szezecinski, A.C.S.F., Fontana, M., Bosenbecker, V.K., Ferri, V.C. \& Do Nascimento, C.O. (2019). Uso de harina de baya de uva en la producción de cookies. 41 st World Congress of Vine and Wine.

Sanahuja, J.A. (2015). De los objetivos del milenio al desarrollo sostenible: Naciones Unidas y las metas globales post-2015. En M. Mesa (Coord.), Focos de tensión, cambio geopolítico y agenda global, anuario 2014-2015. (pp. 49-83). Fundación Cultura de Paz y Ceipaz.

Sandoval, M., Lazarte, K. \& Henao, I. (2008). Hepatoprotección antioxidante de la cáscara y semilla de Vitisvinifera L. (uva). Anales de la Facultad de Medicina, 69 (4), 250-259.

Sato, M., Bagchi, D., Tosaki, A., \& Das, D.K. (2001). Grape seed proanthocyanidin reduces cardiomyocyte apoptosis by inhibithing ischemia/reperfusion-induced activacion of JNK-1 and C-JUN. Free Radical Biology \& Medicine, 31, 729-737. 